\title{
The Effect of Nigella sative Extract on the Fungi that Isolated from Desktop's of the Tables
}

\author{
Duaa Hassan Hadi
}

Technical Institute, Babylon

duaah150@gamil.com

Submission date:- 27/5/2018 Acceptance date:- 11/6/2018 Publication date:- 27/11/2018

Keywords: desktop of the table, Nigella sative, oppertunstic fungi.

\section{Abstract}

The study included the isolation and diagnosis of the fungi from the Desktop's of the tables (instructers ,medical laboratories). The study appeared many of the different kinds of fungi:(Absidia sp,Candidia $s p$, Aspergillus sp,Penicillum sp and Alternaria sp)

The rate of appearance of the type of the fungi in Desktop's of the tables instructers is the higher than the Desktop's of the tables of the medical laboratories .

The rate of appearance of the fungi Candidia is recoded 36.8\% and Penicillum sp,Aspergillus niger is recoded $21.05 \%$ and Aspergillus flavus recoded $5.2 \%$, Abisidia sp recoded $15.7 \%$.

While in the Desktop's of the tables medical laboratories the fungi Aspergillus terrus is recorded $15.7 \%$ and the fungi Absidia,Candidia, Apergillus niger is recoded 20\%, Penicillum sp,Alternaria sp is recoded $10 \%$.

The study showed the influence of the hot water extract of the Nigella sative the concentration which is used $(10,20,30,40) \mathrm{mg} / \mathrm{ml}$ on some of fungi.

The rate of reduction is appeared $100 \%$ of the fungi Penicillum sp in the concentration $(30,40) \mathrm{mg} / \mathrm{ml}$ and the range of the diameter colony of the yeast Candidia albicanis in the concentration $30 \mathrm{mg} / \mathrm{ml}$ the (1) $\mathrm{cm}$.

While the fungi Aspergillus niger in the concentration $(10,20) \mathrm{mg} / \mathrm{ml}$ is given resistance toward the extract 2 .

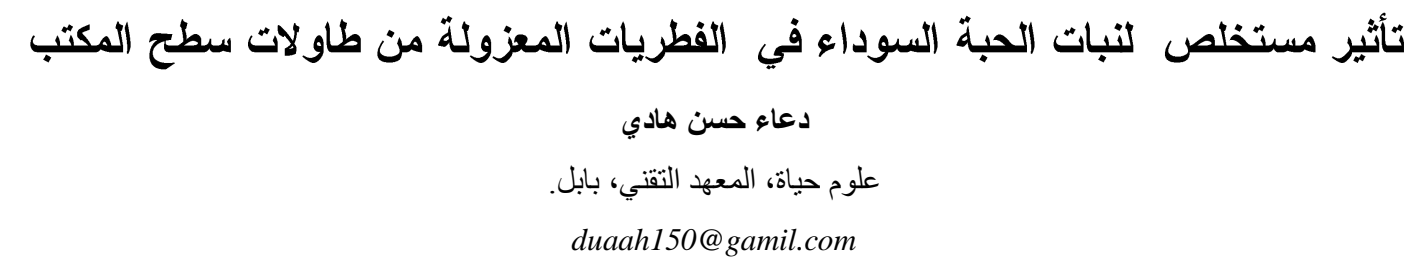

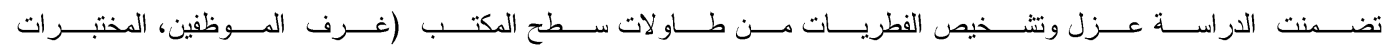

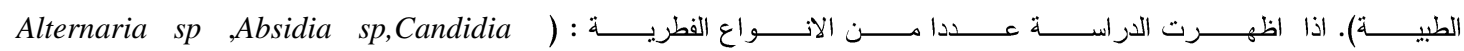

.(sp,Aspergillus sp,Penicillum sp

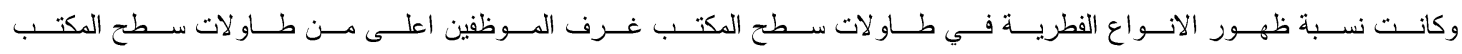

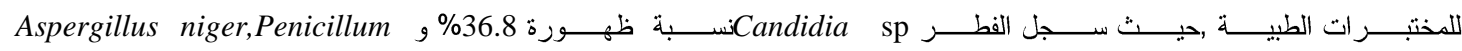

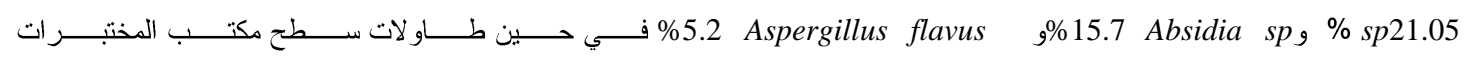

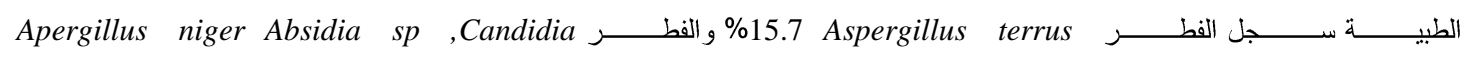

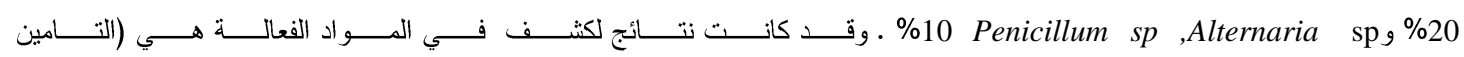




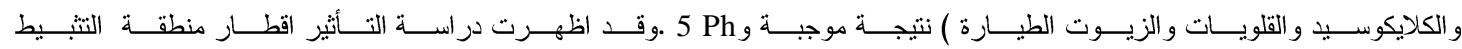

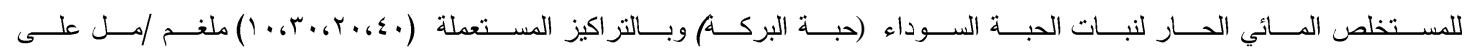

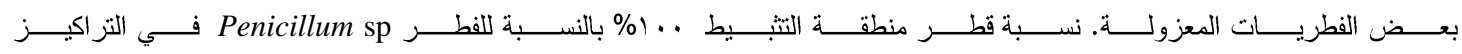

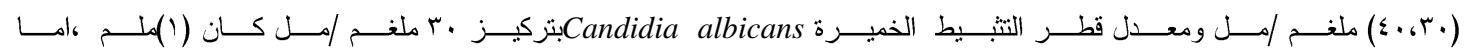

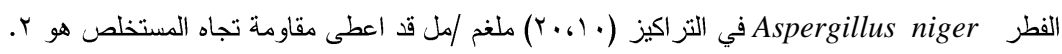
الكلمات الالة: الفطريات الانتهازية، طاو لات سطح المكتب، حبة البركة.

المقدمة:

طاولة مكتب العمل:هي عبارة عن قطعة اثاث التي تستخدم غالبا للجلوس عليها، وقد تصنع طاولة سطح المكتب من لوح زجاجي او خشبي و الذي يوضع عليه الآشياء مثل اقلام، اوراق و (مواد طبية اوكيميائية اذا كان في المختبر). وقد تعد طاولة سطح مكتب العمل هي مصدر بؤرة للجر اثيم من بكتريا وفطريات ،لذلك يجب التخلص من هذه الجر اثيم من خلال استخدام

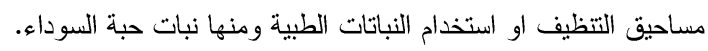
وان للحبة السوداء (Nigella sative) اهمية اقتصادية وطبية وهي عبارة عن عشب نباتي يستخدم كعنصر فعال ومهم في علاج عدد

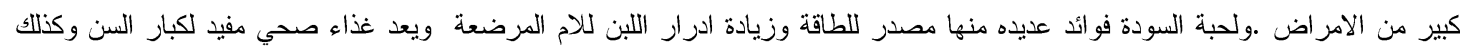

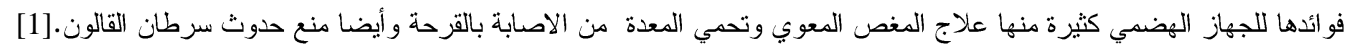

$$
\text { 1- الزيت الطيار :ويكون لها في الغالب التأثير الدوائي. }
$$

ولبذور حبة السوداء تأثير ايجابي على الاسماك ورفع المناعة ومقاومة للأمراض بوكذلك له دور في تخفيف التهاب المفاصل عند

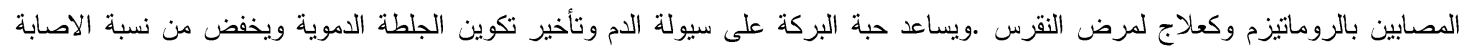
بسرطان الجلد والاورام.ائزم وكعلاج اما التركيب الكيميائي للحبة السوداء فهي تحنوي على ماءبروتين,دهن,كالسيوم,فتامين(A) ,نياسين والياف رماد وسعرات حرارية

وتعد الحبة السوداء غذاء صحيا ومفيدا لكبار السن وذللك لاحتوائها على مواد غذائية متعددة ومنتوعة[4].وان هدف الدراسة هو تحضير المستخلص النباني لبذور حبة البركة بطريقة الاستخلاص المائي الحار فضلا عن كثف العاد المركبات الكيميائية الاساسية.

1- المواد وطر ائق العمل Materials and Methods: ا اعزل الفطريات:

عزلت الاجناس الفطرية من نوعين من طاولات سطح المكتب ( طاولة سطح مكتب غرفة الموظفين، طاولة سطح مكتب المختبر) المصنعه

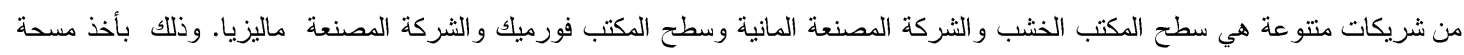

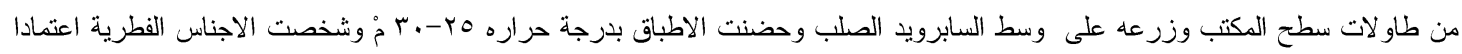

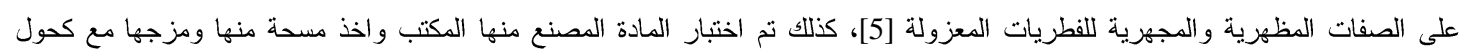
الايثانول بتركيز 90\% ولم يظهر اي نمو فطري[6] ولة

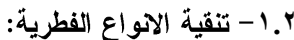

تم تتقية الانواع الفطرية وذلك بنقل اجزاء من المستعمرات النامية المعزولة بشكل اولي بوساطة الناقل الجرثومي المعق (sterial)

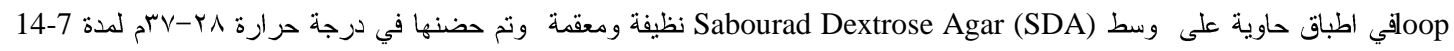

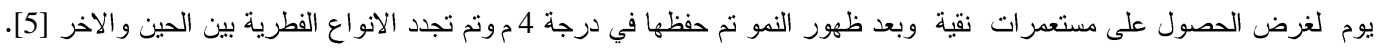




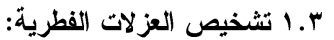

يلاحظ مظهريا معدل نمو مستعمره الفطر في (V-0) ابام عند 0ب-.r مُ على الوسط الزرعي المعقم وتكوين الهايفات الفطرية اما

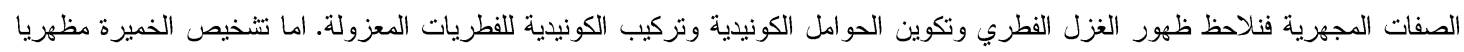
اظهرت مستعمرات محدبة بيضاء صغيرة الحجم على وسط السابرويد اما الصفات المجهرية فضلا عن شكل الخلايا كروية قد تكون متبرعمة

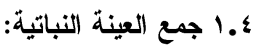

للحصول على العينة النباتية من نبات حبة سوداء لغرض اختبار فاعلية مستخلصها المائية ضد الفطريات المعزولة ,وذللك بثر ائها من الأسواق

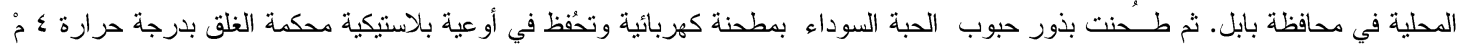
ولحين الاستعمال. 1. 1. 1. تحضير المستخلص المائي الحار:

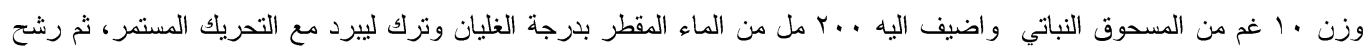

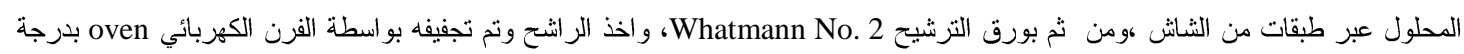
حرارة (.0-0؛) م لحين الحصول على المسحوق المجف ، وكنة ، وكررت هذه العملية لحين الحصول على كمية كافية من المستخلص، جمع المسحوق

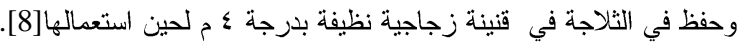

\section{1 اختبار الفعالية التضادية للمستخلص النباتي والمضادات الفطرية في نمو الفطريات قيد الاراسة:}

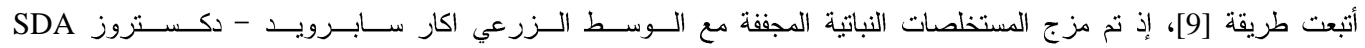

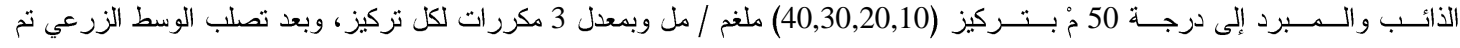

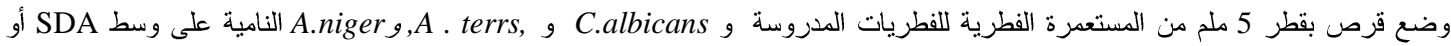

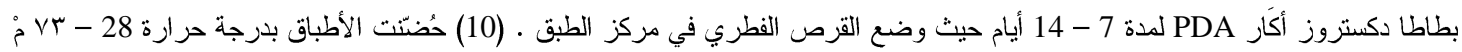
ولمدة 1 - 2 أسابيع ، تم قياس قطر المستعمرة النامية (معدل قطرين متعامدين) وسُّجّلت النتائج.

V. الكثف عن المركبات الفعالة في المستخلص المائي الحار:

اتبعت طريقة [11] للكثف كيمائيا عن المركبات الفعالة للمستخلص المائي الحار لحبة البركة كما في الجدول رقم (1)

جدول (1) الكشف عن المركبات الفعالة الموجودة في نبات حبة البركة

\begin{tabular}{|c|c|}
\hline اسم الكاشف & المركب الكيميائي \\
\hline استعمال محول هيدروكسيد الصوديوم لورقه ترشيح مشبعله & Cuomarin \\
\hline وجود رغوه كثيفه عند رج المستخلص & Saponin \\
\hline قطر ات من kadde بنفسجي & Alkaloid \\
\hline كاثف دراجندروف يعطي لون برتقالي & Glycosid \\
\hline خلات الرصاص مع ظهور راسب هلامي & Tannin \\
\hline ورقه ترشيع مشبعه بلمستخلص وتعرضه لاشعه فوق & Volatile oil \\
\hline
\end{tabular}


r- (التحليل الاحصائي:

حلت نتائج التجارب بحسب التصميم العشو ائي الكامل Completely Randomized Design (C.R. D) ثلاثية العامل وتمت مقارنة

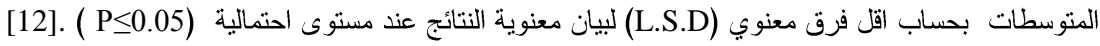

r- النتائج و المناقشة:

تبين في الجدولية (3،2 ان هناك فطريات ظهرت في النوعين كليهما من طاولة سطح المكتب (سطح المكتب للغرفة الموظفين،سطح المكتب

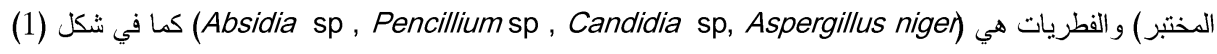

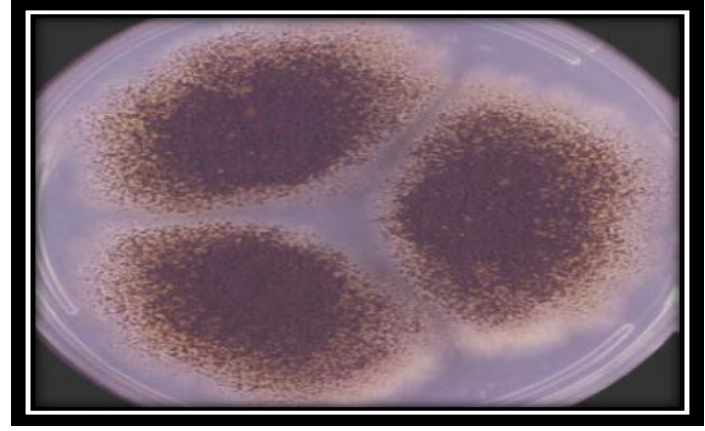

Aspergillus niger $-A$

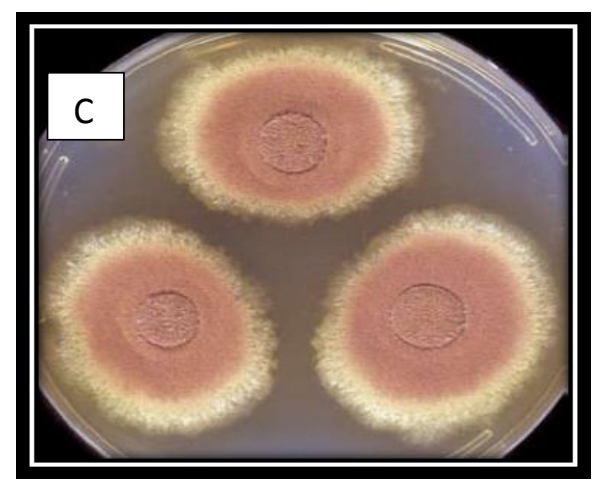

Aspergillus terrus

شكل ( ) يوضح بعض الفطريات المعزولة من طاولات سطح المكتب

Aspergillus niger الفطر -A

Absidia sp الفطر -B

Aspergillus terrus الفطى وسط السابرويد

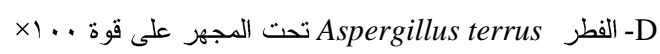


جدول رقم (2) الاجناس الفطرية التي ظهرت في طاولة سطح مكتب الموظفين

\begin{tabular}{|c|c|c|}
\hline النسب المئوية & عدد ظهور الفطر & نوع الفطر \\
\hline 21.05 & 4 & Aspergillus niger \\
\hline 21.05 & 4 & Penicillium sp \\
\hline 15.7 & 3 & Absidia $s p$ \\
\hline 36.8 & 7 & Candidia $s p$ \\
\hline 5.2 & 1 & Aspergillus flavus: \\
\hline
\end{tabular}

جدول رقم (3) الاجناس الفطرية التي ظهرث في طاولة سطح مكتب المختبر

\begin{tabular}{|c|c|c|}
\hline النسب المئوية & عدد ظهور الفطر & نوع الفطر \\
\hline 15.7 & 3 & Aspergillus terrus \\
\hline 20 & 2 & Aspergillius niger \\
\hline 20 & 2 & Candidia albicania \\
\hline 10 & 1 & Sp Alternaria \\
\hline 10 & 1 & Pencillium $s p$ \\
\hline 20 & 2 & Absidia $s p$ \\
\hline
\end{tabular}

ع-اختبار تأثير مستخلص الحبة السوداء المائي على بعض الفطريات المعزولة:

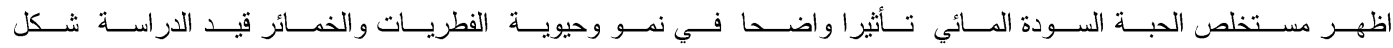

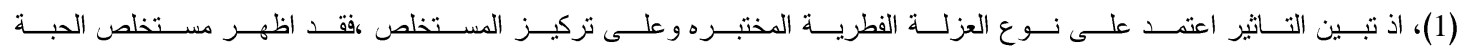

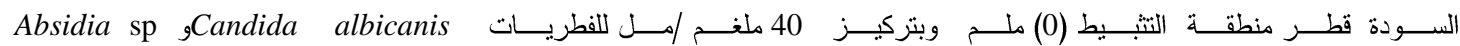

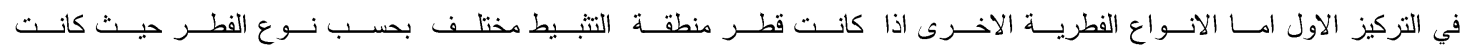

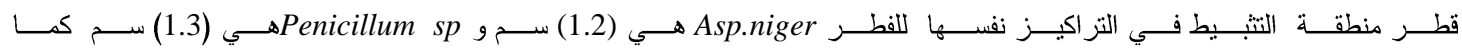
مبين في الجدول الاتي:

جدول رقم (4) بين تاثير مستخلص نبات الحبة السودة المائي على بعض الفطريات المعزولة

\begin{tabular}{|c|c|c|c|c|}
\hline \multicolumn{4}{|c|}{ قطر منطقة التثبيط بالسنتمتر } & \\
\hline 10 & 20 & 30 & 40 & \\
\hline 2.5 & 2 & 1.2 & 1.2 & Aspergillus.niger \\
\hline 1.9 & 1.9 & 1 & 0 & Candida albicans \\
\hline 2.2 & 1.3 & 0 & 0 & Absidia sp \\
\hline 2 & 1.3 & 1.5 & 1.3 & Penicillum sp \\
\hline
\end{tabular}

$\mathrm{LSD}=0.45$

0 - المركبات الفعالة الموجودة في نبات الحبة السوداء :

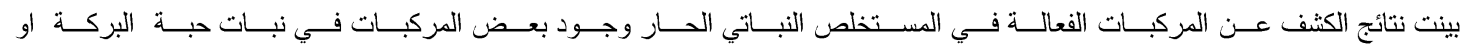

حبة السودة كما موضح في الجدول رقم (5). 
جدول (5) الكثف عن المركبات الكيميائيه في المستخلص المائي الخام لنبات الحبة السوداء:

\begin{tabular}{|c|c|}
\hline النتيجه الفحص & Cuomarin \\
\hline- & Tannin \\
\hline+ & Saponin \\
\hline+ & Alkaloid \\
\hline+ & Glycosid \\
\hline+ & Volatile oil \\
\hline+ & PH \\
\hline 5
\end{tabular}

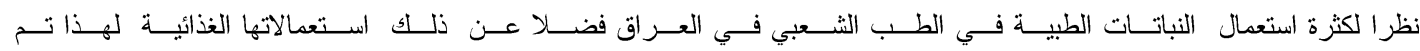

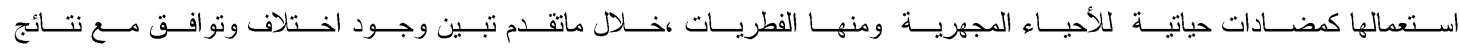

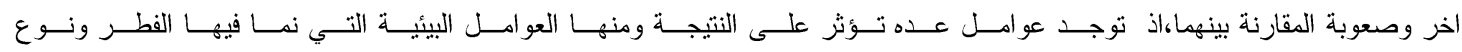

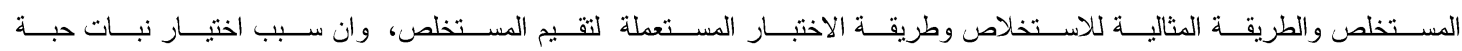

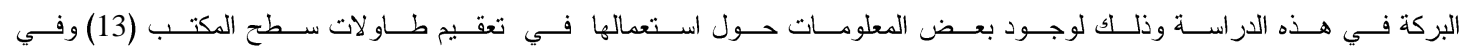

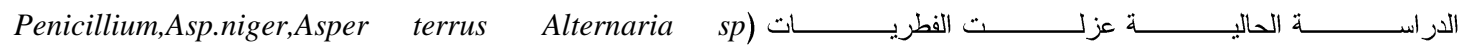

(,Abisidia,Candidia $s p$,

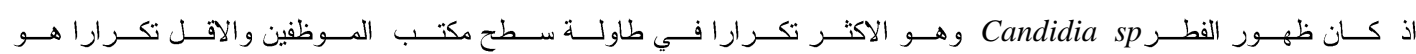

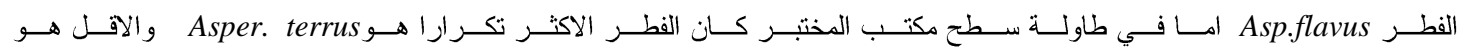

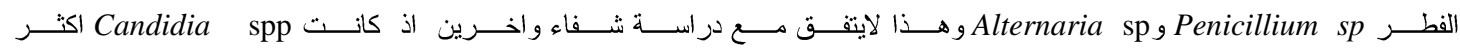

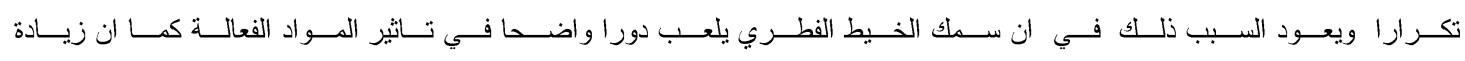

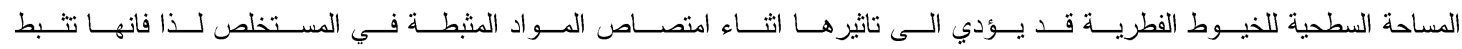
نموها [14]

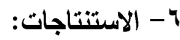

1- يكون المستخلص المائي الحار لبذور الحبة السوداء ذات تاثير عال في تثيط نمو الفطريات وبالتر اكيز العالية.

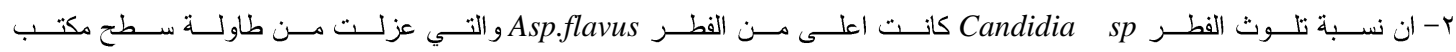

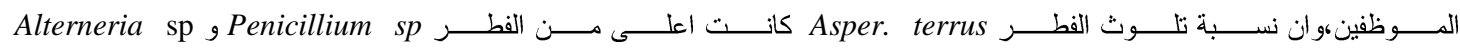
و التي عزلت من طاولة سطح مكتب المختبر.

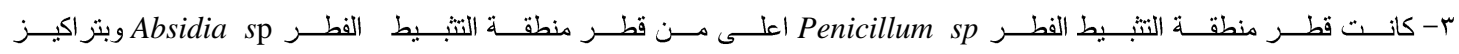
30\%,40\%

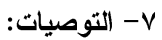

1- تقويم كفاءة تاثير مستخلصات نباتية اخر في تتبيط نمو الفطريات التي تلوث طاو لات سطح المكتب .

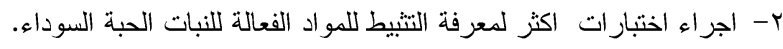


[1] Meral I, Yener Z, Kahraman T, Mert N. "Effect of Nigella sativa on glucose1concentration, lipid peroxidation, anti-oxidant defence system and liver damage in experimentally-induced diabetic rabbits". J Vet Med A Physiol Pathol Clin Med. Dec;48(10):593-9.2001.

[2] Al-Majed AA, Daba MH, Asiri YA, Al-Shabanah OA, Mostafa AA, El-Kashef HA. ."Thymoquinone-induced relaxation of guinea-pig isolated trachea". Res Commun Mol Pathol Pharmacol;110(5-6):333-45. 2001.

[3] Morsi N .M. "Antimicrobial effect of crude extracts of Nigella sativa on multiple antibiotics-resistant bacteria". Acta Microbiol Pol.; 49(1):63-74. 2000.

[4] Mahmood MS, Gilani AH, Khwaja A, Rashid A, Ashfaq MK. "The in vitro effect of aqueous extract of Nigella sativa seeds on nitric oxide production". Phytother Res. Sep;17(8):921-4. 2003.

[5]Kwon-chung,KJ.and Bennett,J.E.” Medical Mycology Keratomycosis 3rded,Williams and Williams and wilkins company",pp105-161.london. 1992.

[6] Hary. A;Walton. R, and Vaishnav .R,. "Environ health composition of eye cosmetics usein Cairo" $J$. Res.14(1)p83-91 Int. 2004.

[7]Peral, G. Carracedo, M.C. Acosta, J. Gallar, J. Pintor. "Increasing levels of diadenosine polyphosphates in dry eye invest". ophthalmol Vis Sci.47 (9):4053. 2006.

$$
\begin{aligned}
& \text { [^]عبد الباقي، انعام عبد المنعم."التأثير التثيطي لعدد من النباتات الطبيه في بعض الجر اثيم المعزوله من الجروح الخمي"، رساله ماجستير،كليه }
\end{aligned}
$$

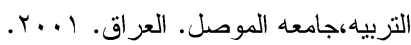

[9] EL-Kady,I.A. ; Mohamed,S.S. \& Mostafa,E.M."Antibacterial and antidermatophyte activities of some essential oils from spices. Qatar". Univ. Sci. J. 13 (1) : 63-69. 1993.

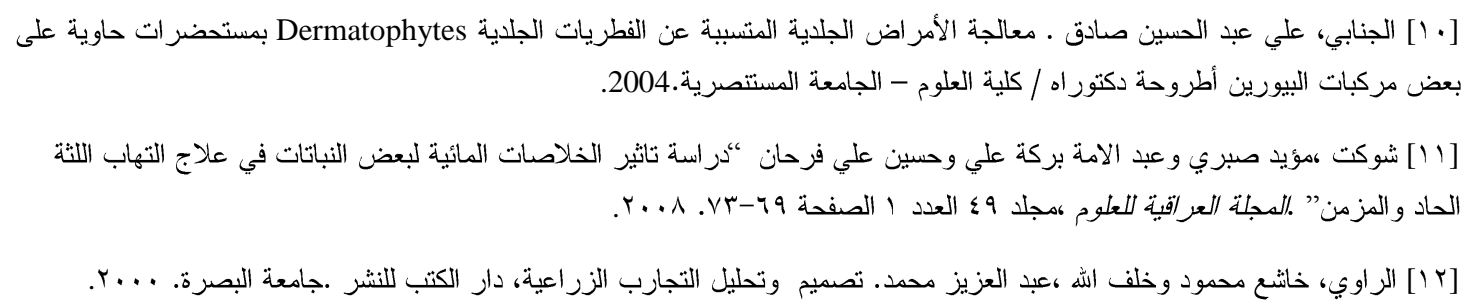

[13] Iddamaldeniya SS, Wickramasinghe N," Thabrew I, Ratnatunge NThammitiyagodage MG. Protection against diethylnitrosoamine-induced hepatocarcinogenesis by an indigenous medicine comprised of Nigella sativa, Hemidesmus indicus and Smilax glabra: a preliminary study”. J Carcinog .Oct 18;2(1):6. 2003.

[14] Ashoor, A. and Abu-Baleer, Y. "Is the Classical Classification of Aspergillosis paranasal sinuses to nonInvasive and Inasive still valid or not?” Bahrain Medical Bulletin. 24 : 91-94. 2002. 